\title{
The Novel Antidepressant Agomelatine Normalizes Hippocampal Neuronal Activity and Promotes Neurogenesis in Chronically Stressed Rats
}

\author{
Girstautè Dagytė $\dot{1}^{1,2}$, Andrea Trentani ${ }^{2}$, Folkert Postema ${ }^{1,2}$, Paul G. Luiten ${ }^{1,2}$, Johan A. Den Boer ${ }^{2}$, \\ Cecilia Gabriel $^{3}$, Elisabeth Mocaër ${ }^{3}$, Peter Meerlo ${ }^{1}$ \& Eddy A. Van der Zee ${ }^{1}$ \\ 1Department of Molecular Neurobiology, University of Groningen, The Netherlands \\ 2Department of Psychiatry, University of Groningen, The Netherlands \\ IInstitut de Recherches Internationales Servier, Courbevoie, France
}

\author{
Keywords \\ Affective disorders; Chronic footshock stress; \\ c-Fos; DCX; Hippocampal cell proliferation; \\ Hippocampal cell survival; HPA-axis.

\section{Correspondence} \\ Girstautè Dagytè, Department of Molecular \\ Neurobiology, University of Groningen, P.O. \\ Box 14, 9750 AA Haren, The Netherlands. \\ Tel.: +3150363 2339; \\ Fax: +31 50363 2331; \\ E-mail: G.Dagyte@rug.nl
}

doi: 10.1111/j.1755-5949.2009.00125.x

Re-use of this article is permitted in accordance with the Creative Commons Deed, Attribution 2.5, which does not permit commercial exploitation.
Agomelatine is a novel antidepressant which acts as a melatonergic (MTl/MT2) receptor agonist and serotonergic $\left(5-\mathrm{HT}_{2 \mathrm{C}}\right)$ receptor antagonist. The antidepressant properties of agomelatine have been demonstrated in animal models as well as in clinical studies. Several preclinical studies reported agomelatine-induced effects on brain plasticity, mainly under basal conditions in healthy animals. Yet, it is important to unravel agomelatine-mediated changes in the brain affected by psychopathology or exposed to conditions that might predispose to mood disorders. Since stress is implicated in the etiology of depression, it is valid to investigate antidepressant-induced effects in animals subjected to chronic stress. In this context, we sought to determine changes in the brain after agomelatine treatment in chronically stressed rats. Adult male rats were subjected to footshock stress and agomelatine treatment for 21 consecutive days. Rats exposed to footshock showed a robust increase in adrenocorticotropic hormone (ACTH) and corticosterone. Chronic agomelatine treatment did not markedly influence this HPA-axis response. Whereas chronic exposure to daily footshock stress reduced cFos expression in the hippocampal dentate gyrus, agomelatine treatment reversed this effect and normalized neuronal activity to basal levels. Moreover, chronic agomelatine administration was associated with enhanced hippocampal cell proliferation and survival in stressed but not in control rats. Furthermore, agomelatine reversed the stress-induced decrease in doublecortin expression in the dentate gyrus. Taken together, these data show a beneficial action of agomelatine in the stress-compromised brain, where it restores stress-affected hippocampal neuronal activity and promotes adult hippocampal neurogenesis.

\section{Introduction}

Affective disorders, such as major depression, represent a serious health problem with long-lasting consequences for both patients and society [1]. Antidepressants, firstly discovered by serendipity, revolutionized the treatment of mood disorders [2]. Yet, more than half a century later, the treatment of these disorders remains a challenge. Only about $50 \%$ of depressed patients treated with currently available medications achieve complete remission [3,4]. Moreover, rather poor tolerability of antidepressant drugs and late onset of their therapeutic effects further increase the risk of unsuccessful treatment [5]. Altogether, this urges the introduction of newer medications with fundamentally different mechanisms of action than the older agents.

Agomelatine is a novel antidepressant which acts as a potent melatonergic (MT1 and MT2) receptor agonist 
[6] and serotonergic $\left(5-\mathrm{HT}_{2 \mathrm{C}}\right)$ receptor antagonist [7]. Current literature indicates that the therapeutic actions of agomelatine are mediated at least partly through different mechanisms than selective serotonin reuptake inhibitors (SSRI) or tricyclics [8]. For example, some of its effects may involve modulation of circadian rhythms and changes in sleep-wake cycle $[9,10]$. The antidepressant properties of agomelatine have been demonstrated both in animal models [11-14] and in clinical studies [15-19]. Yet, the effects of agomelatine merit further investigation.

Beneficial effects of antidepressant drugs are observed in depressed patients but not in healthy volunteers, suggesting that the neurochemical effects of antidepressants might differ in disturbed versus intact systems [5]. Therefore, it is important to unravel agomelatine-mediated changes in the brain affected by psychopathology (clinical studies) or exposed to conditions that might predispose to mood disorders (animal models). It therefore seems a suitable approach to apply agomelatine treatment in chronically stressed animals, in order to reliably explore its beneficial actions and mechanisms behind them.

Stress, especially when severe and prolonged, is one of the major predisposing risk factors for developing depression $[20,21]$. Animal models of chronic stress provide a valuable tool to investigate behavioral, endocrine and neurobiological changes underlying stress-related psychopathologies and mechanism of antidepressant therapies [2,22]. Such models have revealed a variety of stress-induced effects in the brain, such as impaired neurotrophin signaling [23,24], atrophy of hippocampal CA3 pyramidal neurons [25], reduced synaptic plasticity [26], and suppressed neurogenesis in the dentate gyrus $[27,28]$. Together, these changes have been suggested to play a role in the development of neuronal dysfunctions and ultimately increase one's vulnerability to psychopathology [29]. One of the brain areas particularly sensitive to stress is the hippocampus, a region involved in cognition, mood, and neuroendocrine stress reactivity. There is a large body of data supporting the notion that the hippocampus plays a role in depression and the action of antidepressant drugs [30]. The hippocampus is also one of the few regions of the brain where new neurons are generated in adulthood [31,32]. Hippocampal neurogenesis has been established in several different species [33], including primates [34] and humans [35]. During the last decade, numerous studies focused on the hypothesis that changes in hippocampal neurogenesis and plasticity in general contribute to the etiology of depression as well as to the action of antidepressant drugs [36-40]. Preclinical studies suggest that antidepressants may exert their therapeutic effects by enhancing neurotrophin signaling and promoting the formation of new hippocampal neurons as well as new synaptic connections in the brain $[41,42]$.
Such effects have been described for many antidepressant treatments, including serotonin- and norepinephrineenhancing drugs $[43,44]$, electroconvulsive seizures [45], and lithium [46]. In agreement with this, also the novel antidepressant agomelatine promotes hippocampal neurogenesis under basal conditions $[47,48]$.

In the present study, we used a chronic footshock stress model in order to test the effects of agomelatine under conditions of severe and prolonged stress. This footshock model was previously shown to promote HPA-axis hyperactivity, induce robust changes in gene expression in stress-related brain areas, target intracellular signaling cascades involved in neuronal plasticity, and affect hippocampal neurogenesis [49-54]. Here we investigated whether treatment with agomelatine is effective in the brain, exposed to chronic stress. First, we examined whether daily treatment with agomelatine for 21 consecutive days changed HPA-axis reactivity, induced by footshock stress. Then, in order to gain further insight in the processes targeted by stress and antidepressant therapy, we investigated whether agomelatine induced changes in neuronal activity as well as hippocampal neurogenesis.

\section{Experimental Procedures}

\section{Animals and Housing}

The experiments were performed using adult (10-12 weeks of age) male Wistar rats (Harlan, Horst, The Netherlands) weighing 300-350 g at the beginning of the experiment. The animals were housed individually in a climate-controlled room with constant temperature $\left(21 \pm 1{ }^{\circ} \mathrm{C}\right.$ ) and a $12 \mathrm{~h}$ light/ $12 \mathrm{~h}$ dark cycle (lights on at 06:00 h). Food and water was available ad libitum. The experiments were approved by the ethical committee for the use of experimental animals of the University of Groningen and carried out in accordance with the European Communities Council Directive of 24 November 1986 (86/609/EEC).

\section{Experimental Protocol}

Four groups of rats were used for the present experiments: two groups of home-cage control rats (CTR), which were treated either with vehicle (CTR-Veh) or agomelatine (CTR-Ago), and two groups of chronically stressed rats (STR), which were also treated either with vehicle (STR-Veh) or agomelatine (STR-Ago). Each group consisted of 8 animals. The home-cage control rats were sacrificed concomitantly with the stressed rats which were euthanized $24 \mathrm{~h}$ after the last footshock exposure. 


\section{Stress Procedure}

During a 2-week acclimatization period before the start of the experiments, all rats were weighed and handled daily. Footshock stress was applied in a footshock box with a grid floor connected to a shock generator and scrambler $[49,53]$. Stress group rats were subjected to a daily session of footshock stress for 21 consecutive days. During the session in the footshock box rats received 5 uncontrollable and inescapable footshocks $(0.8 \mathrm{~mA}$ in intensity and 8 seconds in duration). All footshock sessions took place during the light phase. In order to increase unpredictability and minimize habituation, both timing and duration of footshock sessions as well as intervals between shocks within a session varied randomly (session starting time: between 08:00 and 13:00 h; session duration: 15-80 min; shock interval: 1-15 min), with the exception of the sessions on days 1,11 , and 21 . On these days, all animals received footshock sessions of 20 min with shock intervals of 5 min to allow standardized neuroendocrine measurements. Control rats stayed undisturbed in their home cages throughout the experiment.

\section{Drug Treatment}

One percent hydroxyethylcellulose (HEC) was used as a vehicle for agomelatine delivery. Agomelatine solution was prepared every day by dissolving agomelatine powder in $1 \%$ HEC at a concentration of $40 \mathrm{mg} / \mathrm{mL}$. The choice of the agomelatine dose was made on the basis of its activity at this concentration in animal models of depression and anxiety [12,55], and on neurogenesis $[47,48]$. Rats were injected intraperitoneally either with agomelatine $(40 \mathrm{mg} / \mathrm{kg})$ or vehicle daily at 16:00 (2 h prior to the dark phase) for 21 days.

\section{Neuroendocrine Measurements}

In order to determine activation of the HPA-axis by footshock stress and changes herein during the experiment, blood samples were collected by tail bleeding and analyzed for adrenocorticotropic hormone (ACTH) and corticosterone [56]. Blood was collected on days 1, 11, and 21 of the chronic stress period. Each time, three samples were taken from all stress group rats: baseline (before the footshock procedure), stress (at the end of the 20-min footshock session), and recovery (30 min after the session in the footshock box). Home-cage control rats were sampled once at the beginning of the light phase, simultaneously with the stress group rats. Blood samples were collected in microcentrifuge tubes containing ethylenediaminetetraacetic acid (EDTA) as the anticoagulant, and kept on ice. Then, blood was centrifuged at $2600 \times g$ for $15 \mathrm{~min}$ and the supernatant stored at $-80^{\circ} \mathrm{C}$ until radioimmunoassay measurements of ACTH and corticosterone were performed.

\section{BrdU Labeling}

In order to study survival of newly born cells in the hippocampus during the experiment, rats received an injection of bromodeoxyuridine (BrdU: Sigma, St. Louis, MO, USA), a synthetic analogue of thymidine which is incorporated into newly synthesized DNA of replicating cells during the $S$ phase of the cell cycle [57]. New cells were labeled 4 days before the start of the experiment with a single intraperitoneal injection $(300 \mathrm{mg} / \mathrm{kg})$. This specific time-point of BrdU labeling for studying cell survival was chosen since it was shown that labeled progenitor cells stop proliferating after 4 days [58]. Such an injection schedule was similarly applied in our laboratory previously [53,59].

\section{Brain Collection and Immunohistochemistry}

At the end of the experiment, $24 \mathrm{~h}$ after the last stress session and $18 \mathrm{~h}$ after the last agomelatine injection, rats were anesthetized with sodium pentobarbital and transcardially perfused with heparinized saline followed by $4 \%$ paraformaldehyde solution in $0.1 \mathrm{M}$ phosphate buffer. Brains were extracted and postfixed in the same solution overnight at $4{ }^{\circ} \mathrm{C}$ and subsequently cryoprotected by immersion in a $30 \%$ buffered sucrose solution for up to $48 \mathrm{~h}$. Coronal serial sections of $30 \mu \mathrm{m}$ were cut using a cryostat and stored in $0.01 \mathrm{M}$ phosphate-buffered saline with $0.1 \%$ sodium azide at $4{ }^{\circ} \mathrm{C}$ until immunohistochemistry was performed.

In order to assess effects of agomelatine and chronic footshock stress on different aspects of hippocampal neurogenesis, immunohistochemistry for Ki-67, doublecortin (DCX), and BrdU was performed. Ki-67 is a nuclear protein which is expressed during all phases of the cell cycle, except G0 [60], which would therefore give an indication of cell proliferation on the last day of the experiment. DCX protein is expressed in newly born cells from 1 day to about 4 weeks of age, and might therefore reveal sustained changes in the neurogenesis process and its modulation $[61,62]$. Finally, since a pool of newborn cells was labeled with an injection of BrdU 4 days prior to the start of the experiment, BrdU immunostaining would indicate changes in survival of new cells in the course of the experiment.

Effects of agomelatine and chronic footshock stress on neuronal activity were assessed by immunohistochemistry for the immediate early gene c-Fos $[63,64]$. 
The immunostainings were performed on free-floating sections under continuous mild agitation. BrdU immunohistochemistry required extra steps for DNA denaturation. For this purpose, sections were exposed to $2 \times$ saline sodium citrate $(2 \times \mathrm{SSC})$ containing $50 \%$ formamide for $30 \mathrm{~min}$ at $65^{\circ} \mathrm{C}$, followed by a rinse with $2 \times \mathrm{SSC}$, incubation with $2 \mathrm{M} \mathrm{HCl}$ for $30 \mathrm{~min}$ at $37^{\circ} \mathrm{C}$ and a washing step with $0.1 \mathrm{M}$ borate buffer. Brain sections for all four immunostainings were preincubated in 3\% normal serum and $0.1 \%$ TritonX-100, and then incubated with one of the following antibodies for $60 \mathrm{~h}$ at $4{ }^{\circ} \mathrm{C}$ : primary mouseanti-Ki-67 (1:200; Monosan, Uden, The Netherlands), goat-anti-DCX (1:1000; Santa Cruz Biotechnology, Santa Cruz, CA, USA), rat-anti-BrdU (1:800; Serotec, Oxford, UK) or rabbit-anti-c-Fos (1:10000; Calbiochem, Darmstadt, Germany). Subsequently, sections were rinsed in $0.01 \mathrm{M}$ Tris-buffered saline and incubated for $2 \mathrm{~h}$ at room temperature with secondary biotinylated goat-anti-mouse, rabbit-anti-goat, donkey-anti-rat or goat-anti-rabbit antibody depending on the primary antibody host (1:500 for all secondary antibodies, Jackson ImmunoResearch, Suffolk, UK). Then, avidin-biotin complex (1:500, Vector ABC kit, Vector Laboratories, Burlingame, CA, USA) was added for $2 \mathrm{~h}$, after which the staining was visualized with $1 \mathrm{mg} / \mathrm{mL}$ diaminobenzidine and $0.003 \% \mathrm{H}_{2} \mathrm{O}_{2}$. Thereafter, sections were rinsed, mounted on slides, dehydrated, and coverslipped for microscopic analysis. Due to technical problems, brain sections of two animals were excluded from DCX and c-Fos immunostainings.

\section{Quantification}

All analyses were performed by an observer blind to the group assignment. Ki-67 and BrdU positive cells were counted in the subgranular zone (SGZ) and granular cell layer (GCL) of the dentate gyrus in every 12 th section of the hippocampus under $500 \times$ magnification. Immunopositive cells that were located one cell diameter away from SGZ were also included in the analysis. The number of immunopositive cells in each section was expressed relative to the length of the dentate gyrus in order to correct for variation in size of the hippocampus along the rostro-caudal axis of the brain. The length of the dentate gyrus was measured by delineating the entire SGZ in each hippocampus. DCX immunoreactivity (ir) in cell bodies and dendrites of the newly generated cells was measured by a previously described method [53]. Briefly, optical density (OD) of DCX expression was measured in the DG and corrected for nonspecific background labeling, which was measured in the corpus callosum. In total, 7-8 sections per animal (Bregma -2.56 to -6.30 , according to the rat brain atlas, Paxinos $\&$ Watson, 2nd edition) were analyzed by delineating a similarly sized area covering the entire width of SGZ and GCL in each hippocampus. Images were taken using a Leica chargedcoupled device digital camera mounted on a microscope (DMIRB, Leica, Cambridge, UK) at $100 \times$ magnification. The OD was expressed in arbitrary units corresponding to gray levels using a Quantimet 550 image analysis system (Leica). In all three stainings, dorsal and ventral hippocampi were analyzed separately. When similar changes in the dorsal and ventral parts of hippocampus were detected, these data were pooled and effects in the entire hippocampus were presented.

c-Fos positive cells were quantified in 3 hippocampal regions of interest (ROI): CAl, CA3 and dentate gyrus (DG). In total, 4-5 sections per animal (Bregma -2.56 to -4.52 , according to the rat brain atlas, Paxinos $\&$ Watson, 2nd edition) were analyzed by counting immunoreactive cell nuclei in each ROI. Images were taken using a Leica charged-coupled device digital camera mounted on a microscope (DMIRB, Leica) at 50× magnification. Positive cell nuclei above the background threshold in each ROI were counted using a Quantimet 550 image analysis system (Leica) and the data were reported as the number of positive cells $/ 0.1 \mathrm{~mm}^{2}$.

\section{Statistics}

Two-way analysis of variance (ANOVA) was used to analyze the effects of stress and agomelatine treatment on hippocampal neurogenesis and neuronal activation. Repeated measures ANOVA was used to analyze the ACTH and corticosterone responses in stress group animals, as well as baseline values in both control and stress group animals across 3 days. Food intake and body weight gain data were analyzed by repeated measures ANOVA. When appropriate, the Fisher LSD test was used for post-hoc analysis.

\section{Results}

\section{Food Intake and Body Weight}

Food intake and body weight were measured from day -4 to day 22 of the experiment. All rats ate and grew at similar rates during the acclimatization period. Chronic footshock stress reduced food intake (repeated measures ANOVA, time $\times$ stress interaction: $F_{(20,560)}=22.02$, $P<0.001)$, whereas agomelatine had no significant effect (Figure 1A). In contrast, body weight gain was strongly reduced by both chronic stress and agomelatine treatment (repeated measures ANOVA, time $\times$ stress interaction: $\mathrm{F}_{(20,560)}=21.51, P<0.001$; time $\times$ drug interaction: $\mathrm{F}_{(20,560)}=9.55, P<0.001$; Figure $\left.1 \mathrm{~B}\right)$. 

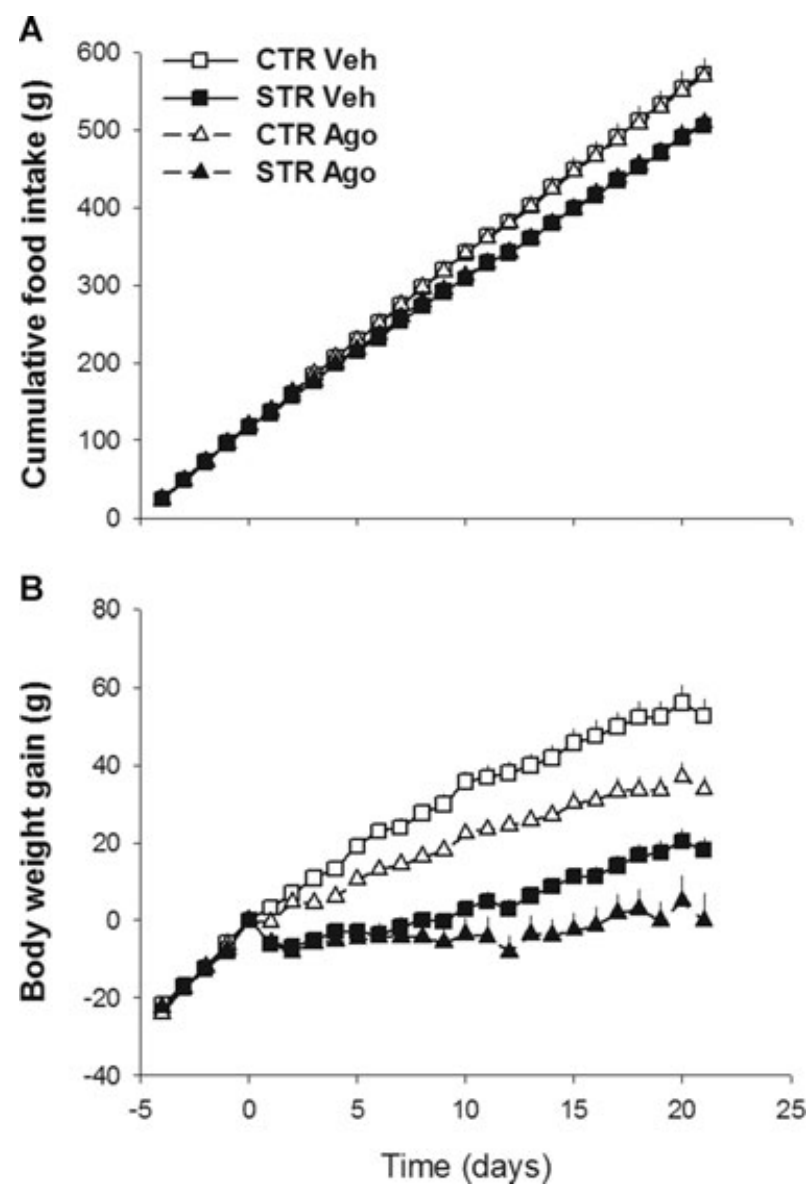

Figure 1 Changes in cumulative food intake and body weight gain in rats subjected to chronic stress and/or agomelatine treatment for 21 days. Exposure to the footshock stress significantly reduced food intake in both rats treated with agomelatine and vehicle (A). Both footshock stress and agomelatine treatment significantly reduced body weight gain (B).

\section{ACTH and Corticosterone Levels}

Changes in plasma ACTH and corticosterone concentrations in response to footshock exposure and agomelatine treatment are shown in Figure 2. Baseline ACTH and corticosterone levels did not differ among the groups. As expected, footshock stress induced a robust HPA-axis activation (repeated measures ANOVA, stress effect: ACTH: $\mathrm{F}_{(8,112)}=91.06, P<0.001$; CORT: $\mathrm{F}_{(8,112)}=111.31$, $P<0.001$; stress vs. baseline: $P<0.001)$. The peak ACTH levels in vehicle-treated animals on day 21 did not differ from the peak ACTH levels on day 1 and day 11 of the experiment. In agomelatine-treated animals, the peak ACTH levels on day 21 were significantly lower as compared to the peak ACTH levels on day l, but not day 11 of the experiment (repeated measures ANOVA, stress $x$ drug interaction: $\mathrm{F}_{(8,112)}=2.39, P=0.02$; day 1 -stress vs. day 21 -stress: $P=0.008$; day 1 -stress vs. day 11 -stress and day 11 -stress vs. day 21 -stress: $P>0.05)$. However, there were no significant differences between the peak ACTH levels of vehicle and agomelatine-treated rats on any of the days tested $(P>0.05)$. There were no differences in corticosterone response between vehicle and agomelatine-treated rats at any time point.

\section{Hippocampal Neuronal Activity}

Figure 3 shows a representative example of c-Fos immunostaining in the hippocampus (A) with a higher magnification of c-Fos-positive cells in the dentate gyrus (B), CA3 (C), and CAl (D) areas. Changes in basal c-Fos expression in hippocampal subregions due to chronic stress and agomelatine treatment are shown in Figure 4. Two-way ANOVA revealed a significant drug effect and a significant stress $x$ drug interaction in cFos-ir in the dentate gyrus $\left(\mathrm{F}_{(1,26)}=5.54, P=0.03\right.$ and $\mathrm{F}_{(1,26)}=13.76, P<0.001$, respectively; CTR Veh: $12.53 \pm$ 1.06; STR Veh: $8.18 \pm 0.74$; CTR Ago: $11.46 \pm 0.91$; STR Ago: $12.97 \pm 0.29$ c-Fos cells $/ 0.1 \mathrm{~mm}^{2}$ ). Post-hoc Fisher LSD test showed a significant chronic stress-induced decrease in c-Fos-ir in vehicle-treated animals (CTR Veh vs. STR Veh: $P<0.001$ ), which was reversed by agomelatine treatment (STR Veh vs. STR Ago: $P<0.001$ ). Although similar changes in c-Fos-ir were noted in CA3 (CTR Veh: $4.58 \pm 0.73$; STR Veh: $2.32 \pm 0.46$; CTR Ago: $4.69 \pm 0.46$; STR Ago: $4.25 \pm 0.96 \mathrm{c}$-Fos cells $/ 0.1 \mathrm{~mm}^{2}$ ) and CAl (CTR Veh: $2.04 \pm 0.66$; STR Veh: $1.14 \pm 0.41$; CTR Ago: $3.18 \pm$ 0.55; STR Ago: $2.47 \pm 0.72 \mathrm{c}$-Fos cells $/ 0.1 \mathrm{~mm}^{2}$ ) areas of hippocampus, they were not statistically significant (drug effect and stress $\times$ drug interaction: $P>0.05$ ).

\section{Hippocampal Neurogenesis}

Figure 5 shows representative examples of Ki-67 (A), BrdU (B), and DCX (C) immunostainings in the hippocampal dentate gyrus. Changes in various stages of hippocampal neurogenesis after chronic stress and agomelatine treatment are depicted in Figure 6. Hippocampal cell proliferation was assessed by Ki-67 immunohistochemistry (Figure 5A). Similar changes in proliferating cell numbers were found in both dorsal (CTR Veh: $4.01 \pm$ 0.35; STR Veh: $3.36 \pm 0.23$; CTR Ago: $4.31 \pm 0.24$; STR Ago: $4.90 \pm 0.29 \mathrm{Ki}-67 \mathrm{cells} / \mathrm{mm})$ and ventral dentate gyrus (CTR Veh: $2.58 \pm 0.23$; STR Veh: $2.16 \pm 0.11$; CTR Ago: $2.72 \pm 0.31$; STR Ago: $3.07 \pm 0.18 \mathrm{Ki}-67$ cells $/ \mathrm{mm}$ ). Therefore, these data were pooled in order to present effects in the entire hippocampus (Figure 6A). Two-way ANOVA revealed a significant drug effect and a significant stress $\times$ drug interaction in proliferating cell numbers $\left(\mathrm{F}_{(1,28)}=10.33, P=0.003\right.$ and $\mathrm{F}_{(1,28)}=5.08, P=$ 0.03, respectively). Post-hoc Fisher LSD test showed that 


\section{Day 1}

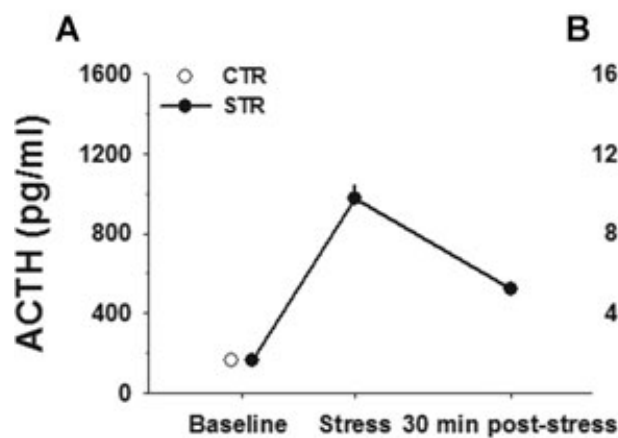

Day 11

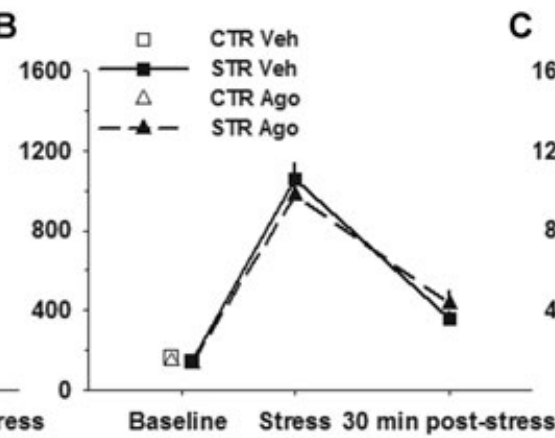

Day 21

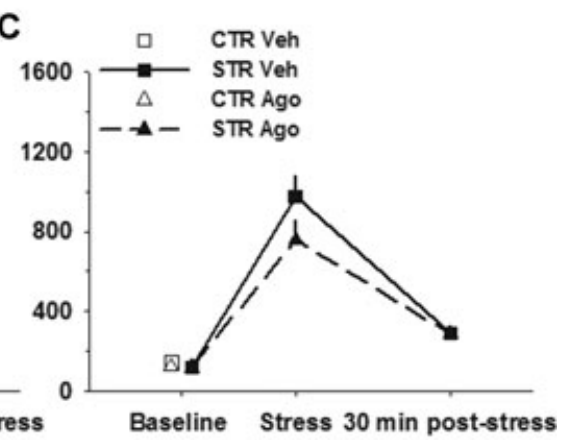

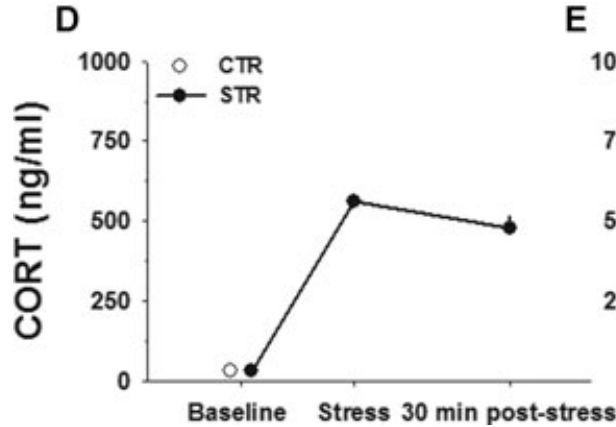

Figure 2 Plasma concentrations of ACTH and corticosterone in response to footshock stress on days 1,11 , and 21 of the experiment. Since on day 1 blood was sampled before the drug treatment started, the data of rats assigned to either vehicle or agomelatine groups were pooled. Exposure to

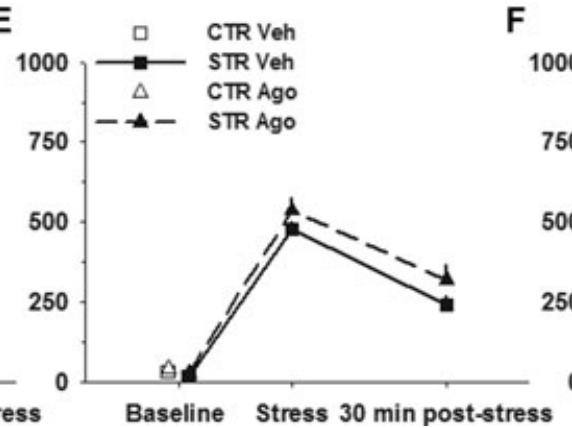

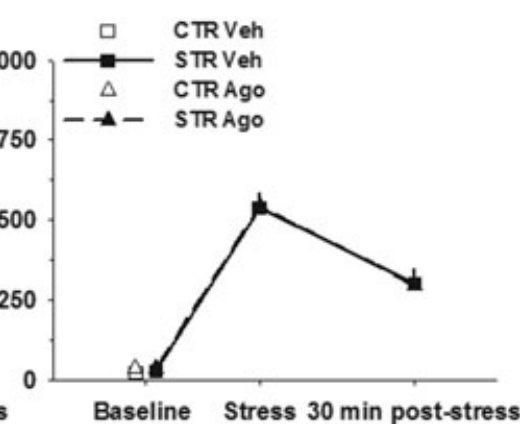

agomelatine significantly increased the number of Ki-67positive cells in stressed animals (STR Veh vs. STR Ago, $P<0.001)$, whereas it had no effect in control animals (CTR Veh vs. CTR Ago, $P>0.05$ ).

Hippocampal cell survival was assessed by BrdU immunohistochemistry (Figure 5B). Whereas no significant effects were observed in the dorsal dentate gyrus (CTR Veh: $4.26 \pm 0.55$; STR Veh: $4.08 \pm 0.51$; CTR Ago: $4.10 \pm 0.33$; STR Ago: $5.50 \pm 0.60 \mathrm{BrdU}$ cells $/ \mathrm{mm}$ ), a significant stress $\times$ drug interaction was found in the ventral dentate gyrus (Figure $6 \mathrm{~B} ; \mathrm{F}_{(1,28)}=4.49, P=0.04$; CTR Veh: $2.92 \pm 0.40$; STR Veh: $2.33 \pm 0.20$; CTR Ago: $2.46 \pm 0.34$; STR Ago: $3.45 \pm 0.50 \mathrm{BrdU}$ cells/mm). Posthoc Fisher LSD test showed that agomelatine significantly increased the number of BrdU-positive cells in stressed animals (STR Veh vs. STR Ago, $P=0.04$ ), whereas it had no effect in control animals (CTR Veh vs. CTR Ago, $P>$ $0.05)$.

Effects of stress and agomelatine on newly born hippocampal cell maturation were assessed by DCX labeling (Figure 5C). Similar changes in DCX expression were the footshock significantly elevated both ACTH and corticosterone levels as compared to baseline values $(P<0.001)$. Chronic agomelatine treatment did not significantly influence either basal or stress-induced HPA-axis activity. found in both dorsal (CTR Veh: 0.31 \pm 0.01; STR Veh: $0.27 \pm 0.01$; CTR Ago: $0.27 \pm 0.01$; STR Ago: $0.30 \pm 0.01$ DCX OD) and ventral dentate gyrus (CTR Veh: $0.32 \pm$ 0.01; STR Veh: $0.28 \pm 0.01$; CTR Ago: $0.28 \pm 0.01$; STR Ago: $0.32 \pm 0.01$ DCX OD). Therefore, these data were pooled in order to present effects in the entire hippocampus (Figure 6C). Two-way ANOVA revealed a significant stress $\times$ drug interaction $\left(\mathrm{F}_{(1,26)}=29.39, P<0.001\right)$. Post-hoc Fisher LSD test showed that both chronic stress and agomelatine reduced DCX expression in the dentate gyrus (CTR Veh vs. STR Veh: $P<0.001$; CTR Veh vs. CTR Ago, $P<0.001)$. In stressed animals, agomelatine reversed the decrease in DCX expression in the dentate gyrus (STR Veh vs. STR Ago: $P=0.001$ ).

\section{Discussion}

Agomelatine administered to chronically stressed rats induced changes in the brain, extending from hippocampal neuronal activity to adult neurogenesis. Whereas chronic exposure to daily footshock stress reduced 


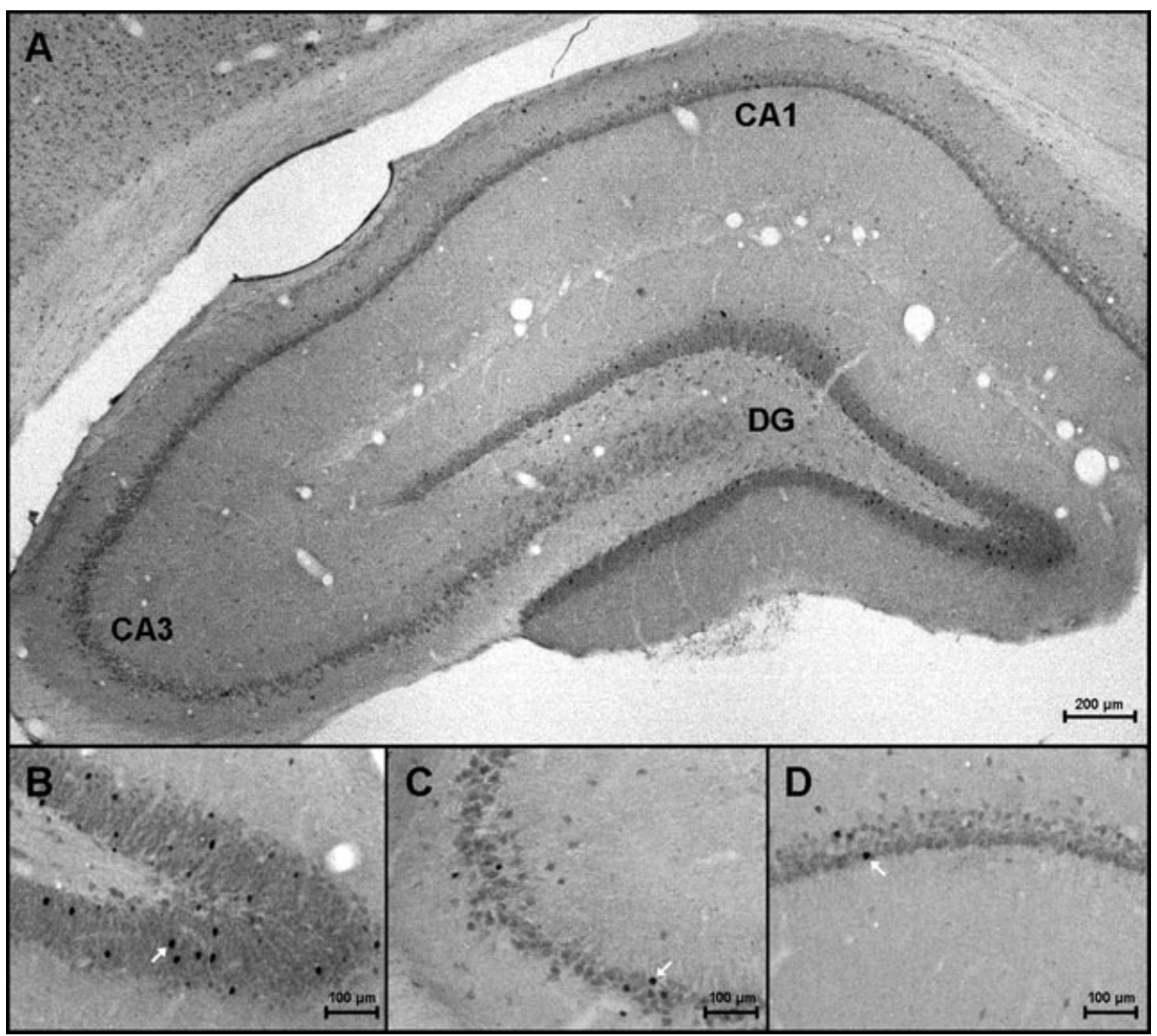

Figure 3 A representative photomicrograph of c-Fos immunostaining in the hippocampus (A). Arrows point at c-Fos-positive cells in the dentate gyrus (B), CA3 (C), and CA1 (D).

c-Fos expression in the dentate gyrus, agomelatine treatment reversed this effect and normalized neuronal activity to basal levels. Chronic agomelatine also enhanced hippocampal cell proliferation in rats concurrently exposed to chronic stress and promoted survival of the newly born cells selectively in the ventral hippocampus. Finally, agomelatine treatment reversed the stressinduced decrease in DCX expression. Taken together, these data show that agomelatine is beneficial in the stress-compromised brain. Our findings suggest that antidepressant action of agomelatine may depend on its ability to reverse stress-affected neuronal activity and promote hippocampal neurogenesis.

Chronic footshock stress was associated with a significant hypophagic effect, confirming the previous reports on reduced body weight gain in stressed male rats $[49,53]$. The 3-week treatment with agomelatine also led to a reduction in growth, although a similar change in body weight in rats of the same strain was not observed in an earlier study after 7-week agomelatine treatment [12]. Reductions in growth were also reported after SSRI administration in rats, in which case it appeared to be a result of decreased food intake $[65,66]$. However, agomelatine administration in our study did not affect food intake. Although agomelatine is not only a potent melatonin receptor agonist but also acts as a $5-\mathrm{HT}_{2 \mathrm{C}}$ antagonist, the decrease in body weight is most likely a melatonin receptor-mediated effect. In rodents, melatonin was reported to decrease body weight without changes in food intake, whereas different $5-\mathrm{HT}_{2 \mathrm{C}}$ antagonists were either shown to increase it or to have no effect $[67,68]$. In contrast to the body weight decrease in rats, agomelatine does not appear to affect body weight in monkeys and humans $[9,69]$.

Footshock stress has been shown to cause a severe activation of the HPA-axis [53]. Pronounced increases in both ACTH and corticosterone were observed here in response to this form of stress. No habituation in the peak 


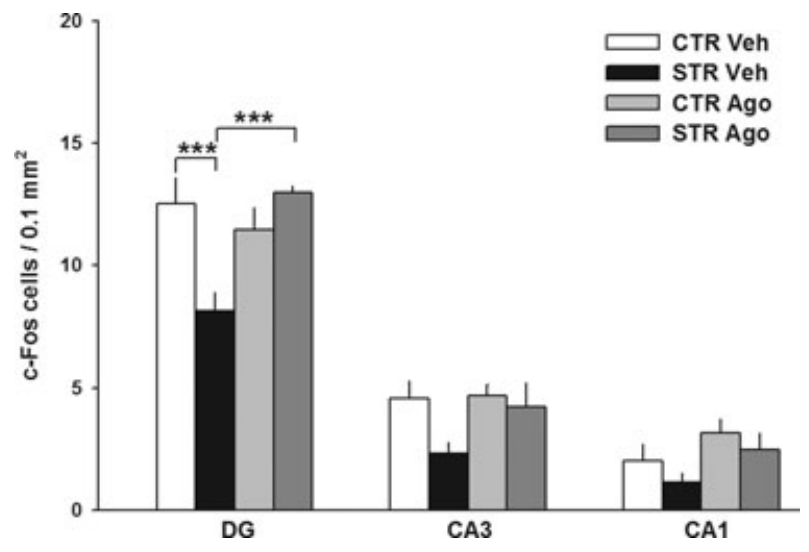

Figure 4 Effects of chronic stress and agomelatine treatment on basal levels of c-Fos expression in hippocampal subregions. In the dentate gyrus, a chronic stress-induced decrease in c-Fos-ir was reversed by agomelatine treatment. Similar effects were noted in CA3 and CA1 regions of the hippocampus, but they did not reach statistical significance. ${ }^{* * *} P<0.001$.

responses was noted, even after 3 weeks of daily exposure to footshock. Agomelatine treatment did not dramatically influence either basal or stress-induced HPA-axis activity. Our data are in line with previous reports where chronic agomelatine had no effect on the HPA-axis parameters after immobilization stress in mice or after predator stress in rats $[13,70]$. On the other hand, this antidepressant has been shown to normalize stress-induced increase in cortisol in the tree shrew model of chronic stress [71]. Differences in the type of stressors and animal species used might be responsible for this inconsistency.

Our c-Fos data suggest that agomelatine acts by normalizing reduced neuronal activity in hippocampus, a brain area sensitive to stress and often implicated in mood disorders. It is interesting to note that whereas several stress-related brain areas display increased c-Fos expression in response to repeated footshock stress, the dentate gyrus of the hippocampus is associated with reduced activation of this immediate early gene $[49,72]$. Here, we observed that such chronic stress-induced suppression of c-Fos expression in the dentate gyrus is prevented by agomelatine treatment. Similar changes were noted in CA3 and CAl regions of the hippocampus, although these did not reach statistical significance. The CA regions showed fewer c-Fos-positive cells than the dentate gyrus, which might have hampered the detection of significant differences between the groups. The c-Fos induction reflects the functional activity of the neurons [64]. Therefore, alterations in neuronal activity may indirectly indicate changes in hippocampal information processing. Although the functional interpretation of c-Fos changes deserves caution, it is tempting to speculate that by normalizing stress-affected neuronal activity in the dentate

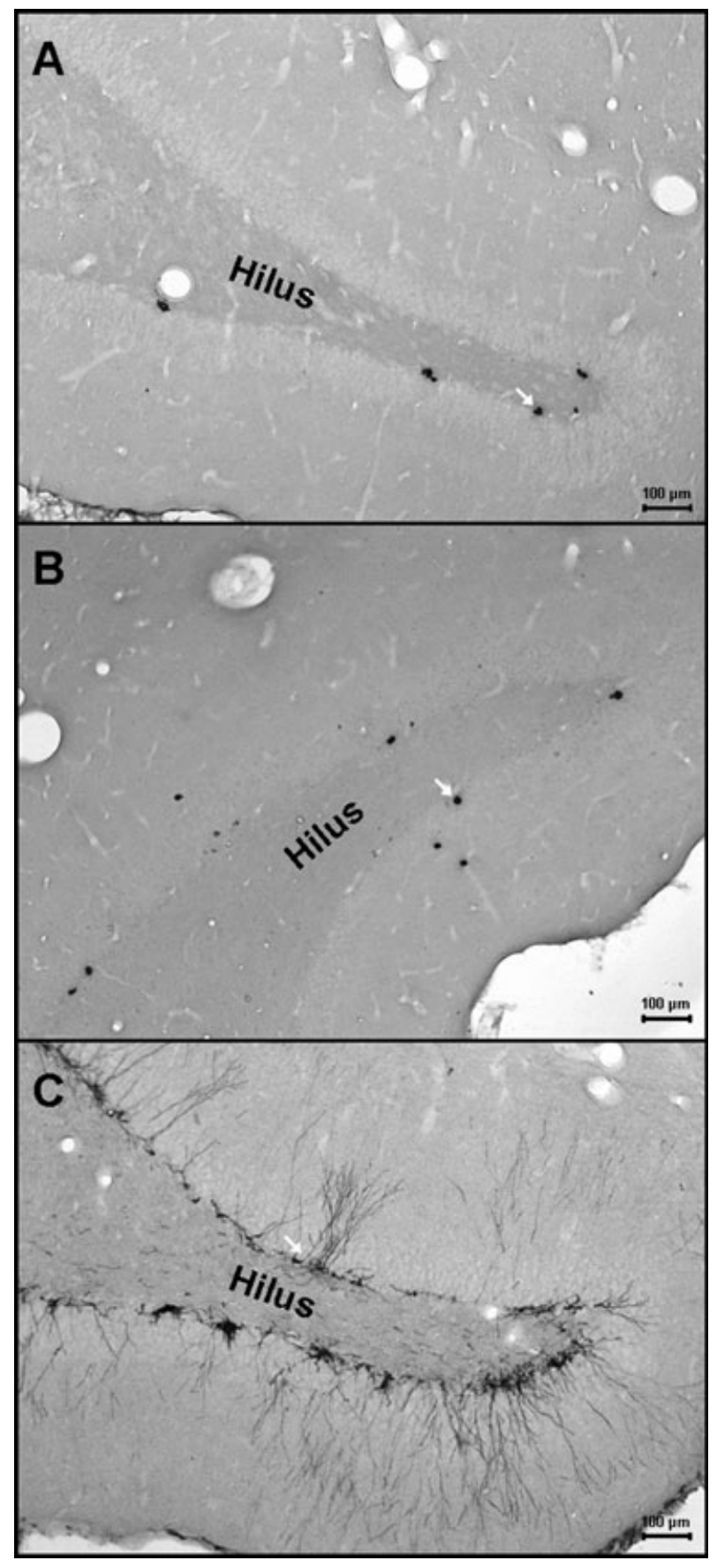

Figure 5 Representative photomicrographs of $\mathrm{Ki}-67(\mathrm{~A}), \mathrm{BrdU}(\mathrm{B})$, and DCX (C) immunostainings in the hippocampal dentate gyrus. Arrows point at Ki-67 (A), BrdU (B), and DCX (C) labeled cells.

gyrus, agomelatine may rescue the functional integrity of hippocampal circuitry.

Prolonged footshock stress did not significantly affect hippocampal cell proliferation or survival in our experiment. The lack of significant stress-mediated effects on these processes may depend on the timing of sacrifice 
A

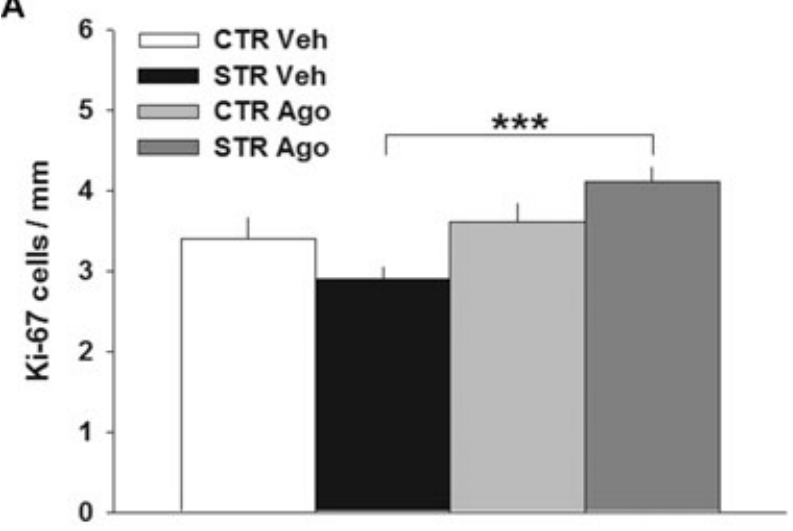

B
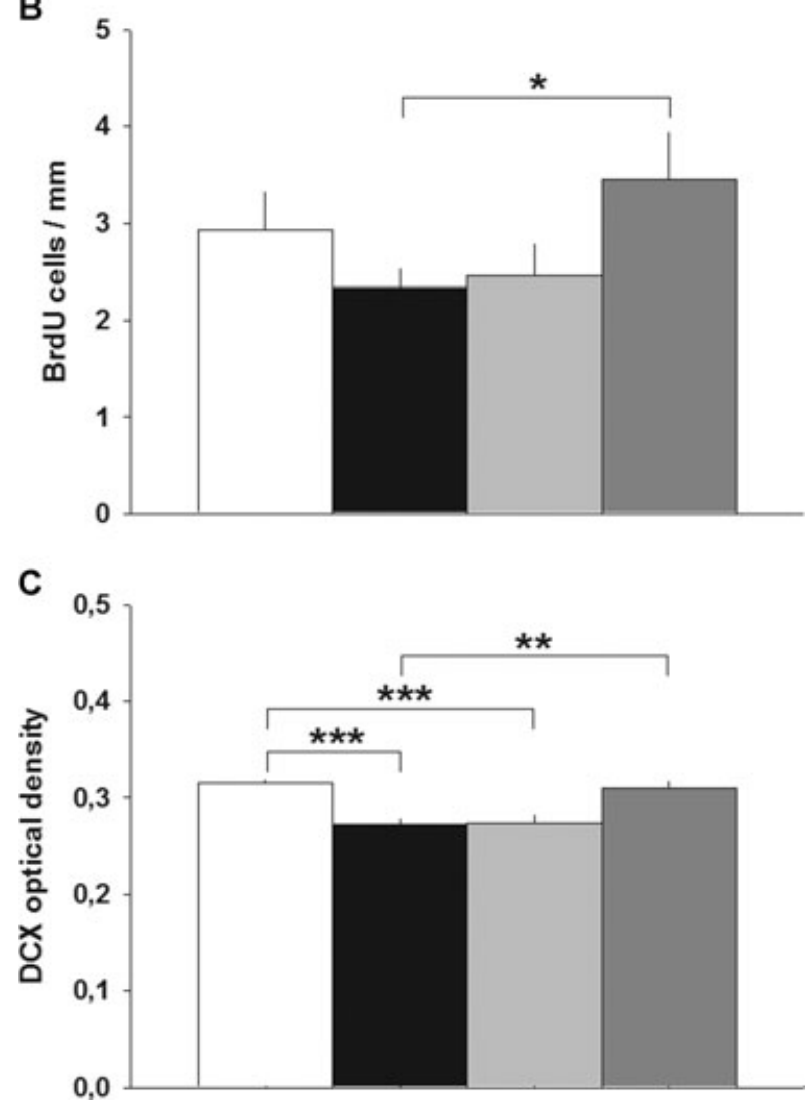

Figure 6 Effects of chronic stress and agomelatine treatment on hippocampal neurogenesis. Agomelatine promoted hippocampal cell proliferation in stressed rats (A). A specific agomelatine-induced effect on cell survival was observed in the ventral hippocampus (B). Both chronic stress and agomelatine therapy reduced DCX expression in the dentate gyrus, whereas a combination of stress and drug treatment reversed this effect (C). ${ }^{*} P<0.05,{ }^{* *} P<0.01,{ }^{* * *} P<0.001$.

of the animals as compared to the last stress session or BrdU labeling. Animals were in fact euthanized $24 \mathrm{~h}$ after the final footshock session. This delay may be sufficient to weaken the inhibitory influences of stress on cell proliferation. A recent study revealed that chronic footshock stress leads to short-lasting suppression of cell proliferation which can easily go unnoticed if animals are sacrificed $24 \mathrm{~h}$ after stress [53]. Interestingly, agomelatine promoted hippocampal cell proliferation and, to a lesser extent, cell survival, in rats subjected to chronic footshock stress. Whereas agomelatine-induced increase in cell proliferation was observed in the entire hippocampus, cell survival was selectively enhanced in the ventral hippocampus, a region particularly involved in response to stress and anxiety [73]. Current literature regarding agomelatine-induced effects on hippocampal neurogenesis is scarce. Yet, it has been shown that 3-week agomelatine treatment increased cell proliferation in the ventral dentate gyrus of non-stressed Wistar rats, whereas extending the treatment for additional 3 weeks also promoted cell survival in the entire dentate gyrus [47]. Agomelatine-mediated effect on hippocampal cell proliferation was recently corroborated by another study, which, in addition, reported that agomelatine administration for 15 or 21 days increased cell survival [48]. However, unpublished data suggest that 6-week agomelatine treatment had no effect on cell survival in non-stressed Sprague-Dawley rats [74]. Notably, our data indicate that chronic agomelatine did not influence hippocampal cell proliferation or survival in non-stressed rats. Discrepant effects among studies may be due to subtle changes in the experimental protocols, such as timing of BrdU injection in relation to agomelatine administration and animal sacrifice. Group-housing conditions (three rats per cage) in the experiments performed by the team of Daszuta might also account for different outcomes in the rates of cell proliferation and survival $[47,48]$. Recently, it has been shown that social isolation affects hippocampal function by decreasing expression of brain-derived neurotrophic factor (BDNF) [75] and preventing or delaying exerciseinduced increase in neurogenesis [76,77]. In view of this, the positive effect of agomelatine on cell proliferation and survival might have been attenuated in our experiment by the individual housing conditions. Nevertheless, agomelatine promoted hippocampal neurogenesis in chronically stressed rats, confirming the previous observations on agomelatine's action in animal models of depression [74,78]. Although the agomelatine-induced stimulation of hippocampal cell proliferation and survival was rather mild, yet, it may indicate a compensatory process, targeted at improving neuronal connectivity in the brain affected by chronic stress.

In order to get more insight into agomelatine-induced changes in hippocampal neurogenesis after chronic stress, we examined the patterns of DCX expression in the dentate gyrus. Chronic footshock stress was associated with a significant decline of DCX expression. Our previous 
study revealed that such a stress-induced reduction in DCX labeling corresponds to a decreased number of DCXpositive immature neurons [53]. Agomelatine also reduced DCX expression, which may depend on its ability to speed up the maturation of newly born cells [48] and shortening in the time window of the expression of this marker [47]. Interestingly, our data demonstrate that such effects on DCX expression are negated when animals are exposed to both chronic stress and agomelatine treatment. Agomelatine reversed the stress-induced reduction in DCX expression, suggesting a beneficial effect of this novel antidepressant on neurogenesis despite the concomitant stressful conditions.

It seems that regulation of DCX-positive cells by stress and agomelatine is executed via different mechanisms. DCX protein is transiently expressed during a period of several weeks that extends from proliferative progenitors to postmitotic neurons $[79,80]$. About $70 \%$ of DCXpositive cells are postmitotic and represent young immature neurons, whereas about $20 \%$ are still in cell cycle and the remaining $10 \%$ are progressing from late neuroblasts to immature neurons [81]. It was proposed that regulation of adult hippocampal neurogenesis primarily occurs during the stages associated with DCX expression [79]. Importantly, we measured DCX reactivity in the entire cell population expressing this protein, extending from proliferating progenitors to immature yet postmitotic neurons. It is possible that stress and antidepressants induce changes only in specific populations of DCX-positive cells. Indeed, a recent study raised the possibility of differential regulation of the distinct populations of DCX cells by pharmacological (chronic escitalopram treatment) and environmental (chronic mild stress) stimuli [82]. In particular, chronic stress was associated with less DCX-expressing postmitotic neurons, whereas escitalopram treatment resulted in an increased number of DCX-positive proliferating progenitors [82]. Earlier, fluoxetine had been reported to cause an increase in the number of DCX-positive neuroblasts [83]. Moreover, chronic fluoxetine also accelerates the maturation of DCX-positive immature neurons, as shown by an increased number of DCX cells with more complex dendritic arborization and an increased proportion of newborn neurons that cease to express DCX marker [84]. Based on these observations, we propose that the chronic stress-induced decrease in the number of DCXexpressing immature neurons is counteracted by agomelatine which promotes the proliferating DCX-positive cells and accelerates their progression toward postmitotic neurons.

In summary, this study elucidated several neurobiological processes targeted by agomelatine treatment in chronically stressed rats. First, agomelatine normalized stress-affected neuronal activity in the dentate gyrus of the hippocampus. Second, agomelatine stimulated hippocampal cell proliferation and survival under conditions of chronic stress. Third, treatment with agomelatine reversed the stress-induced decrease in DCX expression in the dentate gyrus. Taken together, these data indicate that agomelatine is beneficial in the stressed brain, where it preserves the functional activity of hippocampal neuronal circuitry and promotes hippocampal neurogenesis.

\section{Acknowledgments}

This study was supported by a grant from Servier (France). We would like to thank T. Cetin, H. Hulshof, and A. Novati for their help with the stress experiments and blood sampling, J. Bruggink for the ACTH and corticosterone analysis, and J. Keijser for technical assistance with the Quantimet image analysis system.

\section{Conflicts of Interest}

The authors declare no conflict of interests.

\section{References}

1. Greden JF. The burden of recurrent depression: Causes, consequences, and future prospects. J Clin Psychiatry 2001;62 (Suppl 22):5-9.

2. Nestler EJ, Gould E, Manji H, et al. Preclinical models: Status of basic research in depression. Biol Psychiatry 2002;52:503-528.

3. Nestler EJ, Barrot M, DiLeone RJ, Eisch AJ, Gold SJ, Monteggia LM. Neurobiology of depression. Neuron 2002;34:13-25.

4. Fagiolini A, Kupfer DJ. Is treatment-resistant depression a unique subtype of depression? Biol Psychiatry 2003;53:640-648.

5. Berton O, Nestler EJ. New approaches to antidepressant drug discovery: Beyond monoamines. Nat Rev Neurosci 2006;7:137-151.

6. Audinot V, Mailliet F, Lahaye-Brasseur C, et al. New selective ligands of human cloned melatonin MTl and MT2 receptors. Naunyn Schmiedebergs Arch Pharmacol 2003;367:553-561.

7. Millan MJ, Gobert A, Lejeune F, et al. The novel melatonin agonist agomelatine (S20098) is an antagonist at 5-hydroxytryptamine2C receptors, blockade of which enhances the activity of frontocortical dopaminergic and adrenergic pathways. J Pharmacol Exp Ther 2003; 306:954-964.

8. Hanoun N, Mocaer E, Boyer PA, Hamon M, Lanfumey L. Differential effects of the novel antidepressant agomelatine (S 20098) versus fluoxetine on 5-HT1A 
receptors in the rat brain. Neuropharmacology 2004;47:515-526.

9. Kasper S, Hamon M. Beyond the monoaminergic hypothesis: Agomelatine, a new antidepressant with an innovative mechanism of action. World J Biol Psychiatry 2009;10:117-126.

10. Tuma J, Strubbe JH, Mocaer E, Koolhaas JM. Anxiolytic-like action of the antidepressant agomelatine (S 20098) after a social defeat requires the integrity of the SCN. Eur Neuropsychopharmacol 2005; 15:545-555.

11. Bertaina-Anglade V, la Rochelle CD, Boyer PA, Mocaer E. Antidepressant-like effects of agomelatine (S 20098) in the learned helplessness model. Behav Pharmacol 2006;17:703-713.

12. Papp M, Gruca P, Boyer PA, Mocaer E. Effect of agomelatine in the chronic mild stress model of depression in the rat. Neuropsychopharmacology 2003;28:694-703.

13. Barden N, Shink E, Labbe M, Vacher R, Rochford J, Mocaer E. Antidepressant action of agomelatine (S 20098) in a transgenic mouse model. Prog Neuropsychopharmacol Biol Psychiatry 2005;29:908-916.

14. Bourin M, Mocaer E, Porsolt R. Antidepressant-like activity of S 20098 (agomelatine) in the forced swimming test in rodents: Involvement of melatonin and serotonin receptors. J Psychiatry Neurosci 2004;29:126-133.

15. Loo H, Hale A, D'Haenen H. Determination of the dose of agomelatine, a melatoninergic agonist and selective 5-HT(2C) antagonist, in the treatment of major depressive disorder: A placebo-controlled dose range study. Int Clin Psychopharmacol 2002;17:239-247.

16. Pandi-Perumal SR, Srinivasan V, Cardinali DP, Monti MJ. Could agomelatine be the ideal antidepressant? Expert Rev Neurother 2006;6:1595-1608.

17. Kennedy SH, Emsley R. Placebo-controlled trial of agomelatine in the treatment of major depressive disorder. Eur Neuropsychopharmacol 2006;16: 93-100.

18. Olie JP, Kasper S. Efficacy of agomelatine, a MT1/MT2 receptor agonist with 5-HT2C antagonistic properties, in major depressive disorder. Int J Neuropsychopharmacol 2007; 10:661-673.

19. Goodwin GM, Emsley R, Rembry S, Rouillon F. Agomelatine prevents relapse in patients with major depressive disorder without evidence of a discontinuation syndrome: A 24-week randomized, double-blind, placebo-controlled trial. J Clin Psychiatry 2009;70:1128-1137.

20. Kendler KS, Karkowski LM, Prescott CA. Causal relationship between stressful life events and the onset of major depression. Am J Psychiatry 1999;156:837-841.

21. Paykel ES. The evolution of life events research in psychiatry. J Affect Disord 2001;62:141-149.

22. Fuchs E, Flugge G, Ohl F, Lucassen P, VollmannHonsdorf GK, Michaelis T. Psychosocial stress, glucocorticoids, and structural alterations in the tree shrew hippocampus. Physiol Behav 2001;73:285-291.

23. Rasmusson AM, Shi L, Duman R. Downregulation of BDNF mRNA in the hippocampal dentate gyrus after re-exposure to cues previously associated with footshock. Neuropsychopharmacology 2002;27:133-142.

24. Gronli J, Bramham C, Murison R, et al. Chronic mild stress inhibits BDNF protein expression and CREB activation in the dentate gyrus but not in the hippocampus proper. Pharmacol Biochem Behav 2006;85:842-849.

25. Magarinos AM, McEwen BS, Flugge G, Fuchs E. Chronic psychosocial stress causes apical dendritic atrophy of hippocampal CA3 pyramidal neurons in subordinate tree shrews. J Neurosci 1996;16:3534-3540.

26. Joels M, Krugers HJ, Lucassen PJ, Karst H. Corticosteroid effects on cellular physiology of limbic cells. Brain Res 2009; 1293:91-100.

27. Fuchs E, Flugge G. Stress, glucocorticoids and structural plasticity of the hippocampus. Neurosci Biobehav Rev 1998;23:295-300.

28. Gould E, Tanapat P. Stress and hippocampal neurogenesis. Biol Psychiatry 1999;46:1472-1479.

29. Krishnan V, Nestler EJ. The molecular neurobiology of depression. Nature 2008;455:894-902.

30. Alfonso J, Frasch AC, Flugge G. Chronic stress, depression and antidepressants: Effects on gene transcription in the hippocampus. Rev Neurosci 2005; 16:43-56.

31. Abrous DN, Koehl M, Le Moal M. Adult neurogenesis: From precursors to network and physiology. Physiol Rev 2005;85:523-569.

32. Ming GL, Song H. Adult neurogenesis in the mammalian central nervous system. Annu Rev Neurosci 2005;28:223-250.

33. Gould E. How widespread is adult neurogenesis in mammals? Nat Rev Neurosci 2007;8:481-488.

34. Gould E, Reeves AJ, Fallah M, Tanapat P, Gross CG, Fuchs E. Hippocampal neurogenesis in adult Old World primates. Proc Natl Acad Sci U S A 1999;96:5263-5267.

35. Eriksson PS, Perfilieva E, Bjork-Eriksson T, Alborn AM, Nordborg C, Peterson DA, Gage FH. Neurogenesis in the adult human hippocampus. Nat Med 1998;4:1313-1317.

36. Jacobs BL, Praag H, Gage FH. Adult brain neurogenesis and psychiatry: A novel theory of depression. $\mathrm{Mol}$ Psychiatry 2000;5:262-269.

37. Duman RS. Depression: A case of neuronal life and death? Biol Psychiatry 2004;56:140-145.

38. Drew MR, Hen R. Adult hippocampal neurogenesis as target for the treatment of depression. CNS Neurol Disord Drug Targets 2007;6:205-218.

39. Fuchs E, Czeh B, Kole MH, Michaelis T, Lucassen PJ. Alterations of neuroplasticity in depression: The hippocampus and beyond. Eur Neuropsychopharmacol 2004;14(Suppl 5):S481-S490. 
40. Lucassen PJ, Meerlo P, Naylor AS, et al. Regulation of adult neurogenesis by stress, sleep disruption, exercise and inflammation: Implications for depression and antidepressant action. Eur Neuropsychopharmacol 2010;20:1-70.

41. Castren E. Neurotrophins as mediators of drug effects on mood, addiction, and neuroprotection. Mol Neurobiol 2004;29:289-302.

42. Duman RS. Role of neurotrophic factors in the etiology and treatment of mood disorders. Neuromolecular Med 2004;5:11-25.

43. Malberg JE, Eisch AJ, Nestler EJ, Duman RS. Chronic antidepressant treatment increases neurogenesis in adult rat hippocampus. J Neurosci 2000;20:9104-9110.

44. Manev H, Uz T, Smalheiser NR, Manev R. Antidepressants alter cell proliferation in the adult brain in vivo and in neural cultures in vitro. Eur J Pharmacol 2001;411:67-70.

45. Madsen TM, Treschow A, Bengzon J, Bolwig TG, Lindvall $\mathrm{O}$, Tingstrom A. Increased neurogenesis in a model of electroconvulsive therapy. Biol Psychiatry 2000;47:1043-1049.

46. Chen G, Rajkowska G, Du F, Seraji-Bozorgzad N, Manji HK. Enhancement of hippocampal neurogenesis by lithium. J Neurochem 2000;75:1729-1734.

47. Banasr M, Soumier A, Hery M, Mocaer E, Daszuta A. Agomelatine, a new antidepressant, induces regional changes in hippocampal neurogenesis. Biol Psychiatry 2006;59:1087-1096.

48. Soumier A, Banasr M, Lortet S, et al. Mechanisms contributing to the phase-dependent regulation of neurogenesis by the novel antidepressant, agomelatine, in the adult rat hippocampus. Neuropsychopharmacology 2009;34:2390-2403.

49. Trentani A, Kuipers SD, te Meerman GJ, Beekman J, ter Horst GJ, den Boer JA. Immunohistochemical changes induced by repeated footshock stress: Revelations of gender-based differences. Neurobiol Dis 2003;14: 602-618.

50. Trentani A, Kuipers SD, Ter Horst GJ, Den Boer JA. Selective chronic stress-induced in vivo ERK1/2 hyperphosphorylation in medial prefrontocortical dendrites: Implications for stress-related cortical pathology? Eur J Neurosci 2002;15:1681-1691.

51. Kuipers SD, Trentani A, Den Boer JA, Ter Horst GJ. Molecular correlates of impaired prefrontal plasticity in response to chronic stress. J Neurochem 2003;

85:1312-1323.

52. Kuipers SD, Trentani A, Westenbroek C, et al. Unique patterns of FOS, phospho-CREB and BrdU immunoreactivity in the female rat brain following chronic stress and citalopram treatment. Neuropharmacology 2006;50:428-440.

53. Dagyte G, Van der Zee EA, Postema F, Luiten PG, Den Boer JA, Trentani A, Meerlo P. Chronic but not acute foot-shock stress leads to temporary suppression of cell proliferation in rat hippocampus. Neuroscience, 2009; 162:904-913.

54. Westenbroek C, Den Boer JA, Veenhuis M, Ter Horst GJ. Chronic stress and social housing differentially affect neurogenesis in male and female rats. Brain Res Bull 2004;64:303-308.

55. Millan MJ, Brocco M, Gobert A, Dekeyne A. Anxiolytic properties of agomelatine, an antidepressant with melatoninergic and serotonergic properties: Role of 5-HT2C receptor blockade. Psychopharmacology (Berl), 2005; 177:448-458.

56. Meerlo P, Koehl M, van der Borght K, Turek FW. Sleep restriction alters the hypothalamic-pituitary-adrenal response to stress. J Neuroendocrinol 2002;14: 397-402.

57. Christie BR, Cameron HA. Neurogenesis in the adult hippocampus. Hippocampus 2006;16:199-207.

58. Dayer AG, Ford AA, Cleaver KM, Yassaee M, Cameron HA. Short-term and long-term survival of new neurons in the rat dentate gyrus. J Comp Neurol 2003;460: 563-572.

59. Van Der Borght K, Meerlo P, Luiten PG, Eggen BJ, Van der Zee EA. Effects of active shock avoidance learning on hippocampal neurogenesis and plasma levels of corticosterone. Behav Brain Res 2005; 157:23-30.

60. Scholzen T, Gerdes J. The Ki-67 protein: From the known and the unknown. $J$ Cell Physiol 2000;182: 311-322.

61. Rao MS, Shetty AK. Efficacy of doublecortin as a marker to analyse the absolute number and dendritic growth of newly generated neurons in the adult dentate gyrus. Eur J Neurosci 2004; 19:234-246.

62. Brown JP, Couillard-Despres S, Cooper-Kuhn CM, Winkler J, Aigner L, Kuhn HG. Transient expression of doublecortin during adult neurogenesis. J Comp Neurol 2003;467:1-10.

63. Hoffman GE, Lyo D. Anatomical markers of activity in neuroendocrine systems: Are we all 'fos-ed out'? $J$ Neuroendocrinol 2002;14:259-268.

64. Kovacs KJ. c-Fos as a transcription factor: A stressful (re)view from a functional map. Neurochem Int 1998;33:287-297.

65. Wurtman JJ, Wurtman RJ. Fenfluramine and fluoxetine spare protein consumption while suppressing caloric intake by rats. Science 1977;198:1178-1180.

66. Luo SQ, Li ET. Effects of repeated administration of serotonergic agonists on diet selection and body weight in rats. Pharmacol Biochem Behav 1991;38:495-500.

67. Vickers SP, Dourish CT. Serotonin receptor ligands and the treatment of obesity. Curr Opin Investig Drugs 2004;5:377-388.

68. Wolden-Hanson T, Mitton DR, McCants RL, Yellon SM, Wilkinson CW, Matsumoto AM, Rasmussen DD. Daily melatonin administration to middle-aged male rats 
suppresses body weight, intraabdominal adiposity, and plasma leptin and insulin independent of food intake and total body fat. Endocrinology 2000;141: 487-497.

69. Rouillon F. Efficacy and tolerance profile of agomelatine and practical use in depressed patients. Int Clin Psychopharmacol 2006;21(Suppl 1):S31-S35.

70. Conboy L, Tanrikut C, Zoladz PR, et al. The antidepressant agomelatine blocks the adverse effects of stress on memory and enables spatial learning to rapidly increase neural cell adhesion molecule (NCAM) expression in the hippocampus of rats. Int $J$ Neuropsychopharmacol 2009;12:329-341.

71. Fuchs E, Simon M, Schmelting B. Pharmacology of a new antidepressant: Benefit of the implication of the melatonergic system. Int Clin Psychopharmacol 2006;21 (Suppl 1):S17-S20.

72. Huang YH, Cheng CY, Hong CJ, Tsai SJ. Expression of c-Fos-like immunoreactivity in the brain of mice with learned helplessness. Neurosci Lett 2004;363: 280-283.

73. Bannerman DM, Rawlins JN, McHugh SB, et al. Regional dissociations within the hippocampus-memory and anxiety. Neurosci Biobehav Rev 2004;28:273-283.

74. Maccari S, Morley-Fletcher S, Mairesse J, et al. Chronic treatment with agomelatine reversed the decrease in hippocampal cells neurogenesis and survival in prenatally stressed adults rats. Am Soc Neurosci 2005;566.8 (Abstract).

75. Scaccianoce S, Del Bianco P, Paolone G, Caprioli D, Modafferi AM, Nencini P, Badiani A. Social isolation selectively reduces hippocampal brain-derived neurotrophic factor without altering plasma corticosterone. Behav Brain Res 2006; 168: 323-325.
76. Stranahan AM, Khalil D, Gould E. Social isolation delays the positive effects of running on adult neurogenesis. Nat Neurosci 2006;9:526-533.

77. Leasure JL, Decker L. Social isolation prevents exercise-induced proliferation of hippocampal progenitor cells in female rats. Hippocampus 2009;19:907-912.

78. Paizanis E, Renoir T, Lelievre V, et al. Behavioural and neuroplastic effects of the new-generation antidepressant agomelatine compared to fluoxetine in glucocorticoid receptor-impaired mice. Int $J$ Neuropsychopharmacol 2009;24:1-16.

79. Kempermann G, Jessberger S, Steiner B, Kronenberg G. Milestones of neuronal development in the adult hippocampus. Trends Neurosci 2004;27:447-452.

80. Brandt MD, Jessberger S, Steiner B, et al. Transient calretinin expression defines early postmitotic step of neuronal differentiation in adult hippocampal neurogenesis of mice. Mol Cell Neurosci 2003;24:603-613.

81. Plumpe T, Ehninger D, Steiner B, et al. Variability of doublecortin-associated dendrite maturation in adult hippocampal neurogenesis is independent of the regulation of precursor cell proliferation. BMC Neurosci 2006;7:77.

82. Jayatissa MN, Bisgaard CF, West MJ, Wiborg O. The number of granule cells in rat hippocampus is reduced after chronic mild stress and re-established after chronic escitalopram treatment. Neuropharmacology 2008;54:530-541.

83. Encinas JM, Vaahtokari A, Enikolopov G. Fluoxetine targets early progenitor cells in the adult brain. Proc Natl Acad Sci U S A 2006;103:8233-8238.

84. Wang JW, David DJ, Monckton JE, Battaglia F, Hen R. Chronic fluoxetine stimulates maturation and synaptic plasticity of adult-born hippocampal granule cells. J Neurosci 2008;28:1374-1384. 